\title{
SOLAR WIND TURBULENCE FROM MHD TO SUB-ION SCALES: HIGH-RESOLUTION HYBRID SIMULATIONS
}

\author{
Luca Franci ${ }^{1,2}$, Andrea Verdini ${ }^{1,3}$, Lorenzo Matteini ${ }^{4}$, Simone Landi ${ }^{1,5}$, And Petr Hellinger ${ }^{6}$ \\ ${ }^{1}$ Dipartimento di Fisica e Astronomia, Università di Firenze, Largo E. Fermi 2, I-50125 Firenze, Italy \\ ${ }^{2}$ INFN—Sezione di Firenze, Via G. Sansone 1, I-50019 Sesto F.no (Firenze), Italy. \\ ${ }^{3}$ Solar-Terrestrial Center of Excellence-SIDC, Royal Observatory of Belgium, Bruxelles, Belgium. \\ ${ }^{4}$ Department of Physics, Imperial College London, London SW7 2AZ, UK \\ 5 INAF, Osservatorio Astrofisico di Arcetri, Largo E. Fermi 5, I-50125 Firenze, Italy. \\ ${ }^{6}$ Astronomical Institute, AS CR, Bocni II/1401, CZ-14100 Prague, Czech Republic \\ Received 2015 February 12; accepted 2015 April 15; published 2015 May 11
}

\begin{abstract}
We present results from a high-resolution and large-scale hybrid (fluid electrons and particle-in-cell protons) twodimensional numerical simulation of decaying turbulence. Two distinct spectral regions (separated by a smooth break at proton scales) develop with clear power-law scaling, each one occupying about a decade in wavenumbers. The simulation results simultaneously exhibit several properties of the observed solar wind fluctuations: spectral indices of the magnetic, kinetic, and residual energy spectra in the magnetohydrodynamic (MHD) inertial range along with a flattening of the electric field spectrum, an increase in magnetic compressibility, and a strong coupling of the cascade with the density and the parallel component of the magnetic fluctuations at sub-proton scales. Our findings support the interpretation that in the solar wind, large-scale MHD fluctuations naturally evolve beyond proton scales into a turbulent regime that is governed by the generalized Ohm's law.
\end{abstract}

Key words: magnetohydrodynamics (MHD) - plasmas - solar wind - Sun: general - turbulence

\section{INTRODUCTION}

In situ measurements of the solar wind plasma and electromagnetic field show spectra with a power-law scaling spanning several decades in frequency, $f$ (e.g., Alexandrova et al. 2009; Roberts 2010; Sahraoui et al. 2010). Power laws support an interpretation in terms of turbulent fluctuations, although the rich variety of spectral features is not easily explained in the framework of known turbulent theories and phenomenologies.

For frequencies in the so-called magnetohydrodynamic (MHD) range, $10^{-4} \mathrm{~Hz} \lesssim f \lesssim 10^{-2} \mathrm{~Hz}$ at $1 \mathrm{AU}$, the magnetic field spectrum and the kinetic field spectrum have a different scaling, the former being proportional to $f^{-5 / 3}$, while the latter is proportional to $f^{-3 / 2}$ (Podesta et al. 2007; Salem et al. 2009; Tessein et al. 2009; Wicks et al. 2011). While a magnetic excess is generally found in solar wind turbulence, only recently the spectrum of residual energy (the difference between magnetic and kinetic energy) was shown to have a power-law scaling with a spectral index of -2 (Chen et al. 2013a). Such a finding confirms early predictions on the residual energy spectrum (Grappin et al. 1983) and the numerical results of incompressible MHD simulations (Muller \& Grappin 2005). Note that the three spectral indices $(-3 / 2,-5 / 3,-2)$ for the kinetic, magnetic, and residual energy spectrum are not reproduced simultaneously in any direct numerical simulation (DNS; e.g., Muller \& Grappin 2005; Chen et al. 2011b) unless a particular driving is applied to large scales (Boldyrev et al. 2011). Finally, in the MHD range, magnetic and velocity fluctuations are dominated by the transverse components with respect to the ambient magnetic field $\boldsymbol{B}_{0}$ (e.g., Smith et al. 2006; Wicks et al. 2011).

Moving to higher frequencies, $f \gtrsim 10^{-2} \mathrm{~Hz}$, there is growing evidence that kinetic effects become important and change the nature of the self-similar spectra of fluctuations observed for $f \gtrsim 1 \mathrm{~Hz}$. A spectral break appears in magnetic and velocity spectra at proton scales, separating the MHD inertial range cascade from a second power-law interval at kinetic scales. The physical scale associated with this spectral break has not yet been identified (e.g., Bourouaine et al. 2012; Bruno \& Trenchi 2014; Chen et al. 2014). The spectral index of magnetic fluctuations after the break varies between $(-4)$ and $(-2)$ (Leamon et al. 1998; Smith et al. 2006), although it tends to cluster around a slope of -2.8 for higher frequencies (Alexandrova et al. 2012). The change in the turbulence regimes also shows up in the density spectrum (Chen et al. 2013b), which steepens and couples to the parallel component of the magnetic field. The latter becomes as energetic as the two perpendicular components, resulting in an increase of the so-called magnetic compressibility (Alexandrova et al. 2008; Salem et al. 2012; Kiyani et al. 2013). Finally, measurements at $1 \mathrm{AU}$ show that the spectrum of the electric field flattens at about $1 \mathrm{~Hz}$ (Bale et al. 2005; Kellogg et al. 2006), although the noise level hinders the determination of a precise spectral scaling.

The measurement of third-order structure functions at MHD scales (Sorriso-Valvo et al. 2007; MacBride et al. 2008) and of high-order structure functions at MHD (Salem et al. 2009) and sub-proton scales (Kiyani et al. 2013) provided additional evidence that fluctuations are turbulent all the way down to electron scales in the solar wind. While DNSs are able to reproduce some aspects of either the MHD range (e.g., Maron \& Goldreich 2001; Mason et al. 2008; Beresnyak \& Lazarian 2009; Grappin \& Muller 2010; Lee et al. 2010; Boldyrev et al. 2011; Chen et al. 2011b; Dong et al. 2014) or the sub-proton range (e.g., Matthaeus et al. 2008; Camporeale \& Burgess 2011; Howes et al. 2011; Markovskii \& Vasquez 2011; Gary et al. 2012; Servidio et al. 2012; Wan et al. 2012; Boldyrev et al. 2013; Meyrand \& Galtier 2013; Passot et al. 2014), to our knowledge, a clear indication that a turbulent regime is established in the whole spectrum spanning the two ranges has not been reported so far. 
In this work, we present results from a high-resolution hybrid (fluid electrons, particle-in-cell protons) two-dimensional (2D) DNS of turbulence and provide the first direct numerical evidence of the simultaneous occurrence of several features observed in the solar wind spectra. These include (i) the different scaling of magnetic and kinetic fluctuations in the MHD range, (ii) a magnetic spectrum with a clear double power-law scaling separated by a break, (iii) an increase in magnetic compressibility at small scales, and (iv) a strong coupling between density and magnetic fluctuations at small scales. The electric field spectrum is also consistent with observations, showing a change in the spectral properties at sub-proton scales. Our results indicate that the switch in the spectral slopes observed in the solar wind results from the natural continuation of a large-scale MHD turbulent cascade through proton and down to electron scales, where the different field couplings are governed by the non-ideal terms of Ohm's law.

\section{NUMERICAL SETUP}

The kinetic model uses the hybrid approximation: electrons are considered to be a massless, charge neutralizing, isothermal fluid; ions are described by a particle-in-cell model (see Matthews 1994 for detailed model equations). The characteristic temporal and spatial units used in the model are the inverse proton gyrofrequency $1 / \Omega_{p}$ and the proton inertial length $d_{p}=v_{\mathrm{A}} / \Omega_{p}$, respectively, $v_{\mathrm{A}}$ being the Alfvén speed. We use a spatial resolution of $\Delta x=\Delta y=0.125 d_{p}$, and there are 8000 particles per cell $(\mathrm{ppc})$ representing protons. The resistive coefficient is set to the value $\eta=510^{-4} 4 \pi v_{\mathrm{A}} c^{-1} \Omega_{p}^{-1}$ to prevent the accumulation of magnetic energy at the smallest scales. Fields and moments are defined on a 2D $x-y$ grid with dimensions of $2048^{2}$ and with periodic boundary conditions. Protons are advanced with a time step of $\Delta t=0.025 \Omega_{p}^{-1}$, while the magnetic field $\boldsymbol{B}$ is advanced with a smaller time step of $\Delta t_{B}=\Delta t / 10$. The number density $n$ is assumed to be equal for protons and electrons, $n_{p}=n_{e}=n$, and both protons and electrons are isotropic, with $\beta_{p}=\beta_{e}=0.5$ where $\beta_{p, e}=8 \pi n K_{B} T_{p, e} / B_{0}^{2}$ are the proton (electron) betas (here, $K_{B}$ is the Boltzmann's constant, $B_{0}$ the ambient magnetic field, and $T_{p, e}$ are the proton and electron temperatures).

We impose an initial ambient magnetic field of $\boldsymbol{B}_{0}=B_{0} \hat{z}$ perpendicular to the simulation plane. We add an initial spectrum of linearly polarized magnetic and bulk velocity fluctuations $\boldsymbol{u}$, with in-plane components only. Fourier modes of equal amplitude and random phases are excited in the range $-0.2<k_{x, y}<0.2$, assuring energy equipartition and vanishing correlation between kinetic and magnetic fluctuations. Initial velocity fluctuations have vanishing divergence, and density fluctuations are also vanishing (in the limit of numerical noise). Quantities are defined as parallel $(\|)$ and perpendicular $(\perp)$ with respect to $\boldsymbol{B}_{0}$. We define the omnidirectional spectra,

$$
E_{\Psi}\left(k_{\perp}\right) \equiv \delta \Psi^{2}\left(k_{\perp}\right) / k_{\perp}=\sum_{\left|k_{\perp}\right|=k_{\perp}} \hat{\Psi}_{2 \mathrm{D}}^{2}\left(\boldsymbol{k}_{\perp}\right),
$$

where $\hat{\Psi}$ are the Fourier coefficients of a given quantity $\Psi$ (we use $\boldsymbol{E}$ and $\boldsymbol{J}$ to indicate electric field and current density, respectively) and $\delta \Psi\left(k_{\perp}\right)$ is the amplitude of the fluctuation $\Psi$

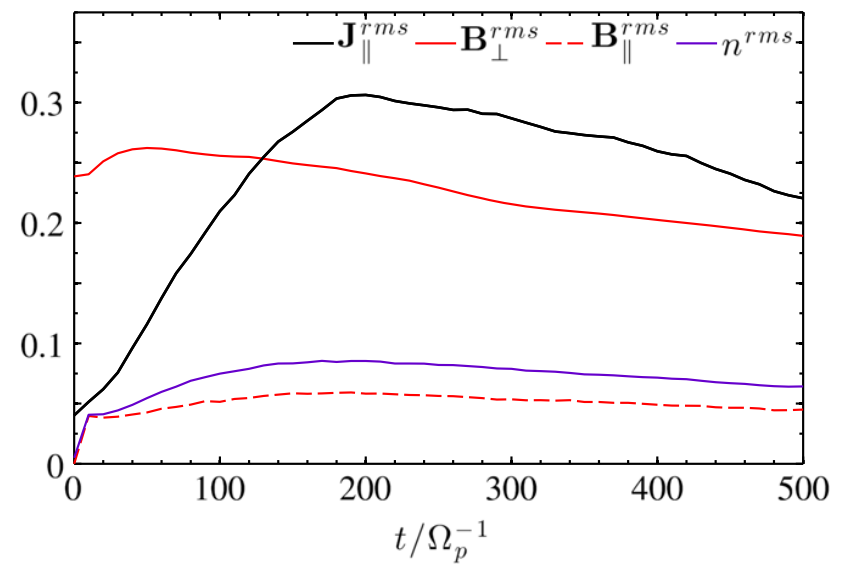

Figure 1. rms parallel current density (black line), perpendicular and parallel magnetic fluctuations (red solid line and red dashed line, respectively), and density fluctuations (purple line) as a function of time (normalized to the inverse of the proton gyrofrequency $\Omega_{p}$ ). As a reference, the nonlinear time at the initial time is about $20 \Omega_{p}^{-1}$.

at the scale $k_{\perp}$. We also define the rms value as

$$
\Psi^{\mathrm{rms}}=\sqrt{\left\langle\Psi^{2}\right\rangle-\langle\Psi\rangle^{2}},
$$

where $\langle\ldots\rangle$ stands for a real-space average over the whole simulation domain. With these definitions, the initial conditions have $E_{u} \sim E_{B} \propto k_{\perp}$, with $B^{\mathrm{rms}} / B_{0} \sim 0.24$ allowing for fast turbulent dynamics sustained for about $300 \Omega_{p}^{-1}$ (the nominal nonlinear time at the beginning of the simulation is approximately $20 \Omega_{p}^{-1}$, but it increases at later time).

\section{RESULTS}

In Figure 1, we plot the rms of the parallel current density, of the parallel and perpendicular magnetic field, and of the density fluctuations. The current density increases until $t=200 \Omega_{p}^{-1}$, reflecting the formation of small scales due to the development of a turbulent cascade, and then declines smoothly. The decay is slow since larger and larger scales continue to feed the cascade at later times. Accordingly, the perpendicular magnetic field declines steadily after a transient increase. Shortly after the beginning, fluctuations in the parallel component of the magnetic field and in the density appear; slowly increase, reaching a shallow maximum at the same time of the current density; and then decline slowly. The initial growth is due to the generation of a low level of compressive fluctuations. Velocity fluctuations (not shown) behave similarly to magnetic fluctuations, with the perpendicular component declining monotonically (there is no initial growth) and the parallel component originating from compressive effects. In the following, we will show spectra at the time of the peak of the current density $t=200 \Omega_{p}^{-1}$, but all the turbulent properties are stable and remain valid until the end of the simulation $\left(t=500 \Omega_{p}^{-1}\right)$.

In Figure 2, we show isocontours of the perpendicular magnetic field energy. This snapshot highlights the formation of intense vortex-like and filamentary structures. The latter reflects the local anisotropy of small-scale fluctuations, while their random orientation assures the statistical isotropy of the 2D spectrum; we thus consider only omnidirectional spectra in the following. 


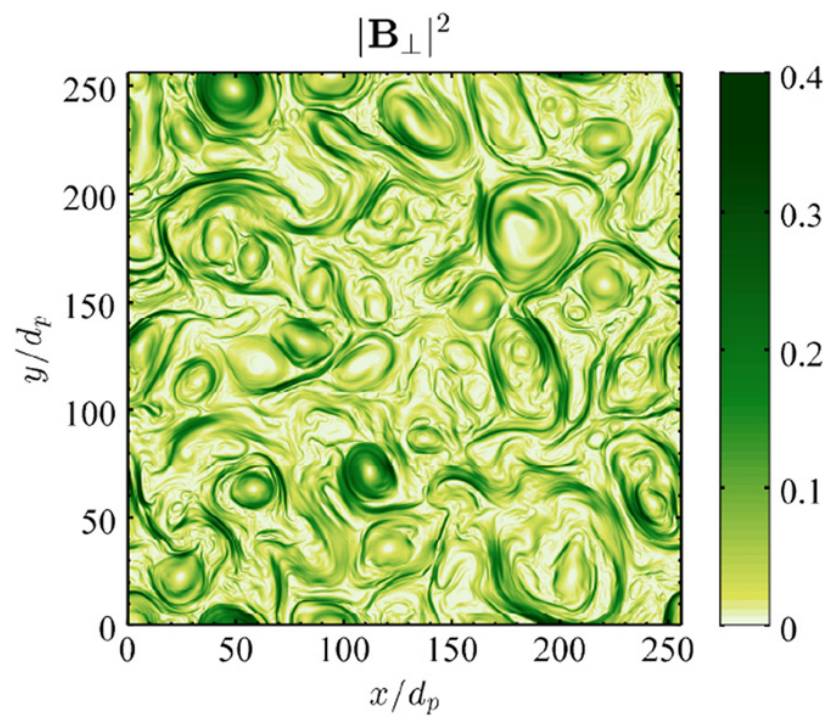

Figure 2. Contour plot of the perpendicular magnetic energy at $t=200 \Omega_{p}^{-1}$.

In Figure 3 (top panel), we show the spectra of the total velocity, magnetic, and electric fields. The magnetic spectrum (red line) has a double power-law scaling, each power-law range occupying about one decade, with a break at $k_{\perp} d_{p} \sim 2$ that separates the MHD from the sub-proton range. The bulk velocity spectrum (blue line) also has a power-law scaling in the MHD range, but it falls off abruptly at $k_{\perp} d_{p} \sim 1$, not showing any clear power law at higher wavenumbers. At smaller scales, it reaches the ppc noise level, estimated as the level of velocity fluctuations at $t=0$ (light blue dashed line). Finally, the electric field spectrum (green line) follows the velocity in the MHD range $\left(k_{\perp} d_{p} \lesssim 0.4\right)$ and tends to flatten as it enters the sub-proton range $\left(k_{\perp} d_{p} \gtrsim 2\right)$.

These spectral properties are qualitatively and quantitatively in agreement with observed solar wind spectra. In the MHD range, the magnetic and kinetic spectra are power laws with scaling consistent with $E_{B} \propto k_{\perp}^{-5 / 3}$ and $E_{u} \propto k_{\perp}^{-3 / 2}$, respectively, as can be seen in the bottom panel of Figure 3 where the spectra are compensated by $k^{5 / 3}$ and $k^{3 / 2}$, respectively. In the same panel, we also plot the residual energy spectrum, $E_{R}=E_{B}-E_{u}$, which has a power-law scaling over about one decade in the MHD range with a spectral index $\approx-2$ as in the observations (Chen et al. 2013a). In addition, in the subproton range the magnetic spectrum scales as $E_{B} \propto k_{\perp}^{-3}$, a spectral index that is very close to the value -2.8 reported in the observations (Alexandrova et al. 2009). Note that the electric field spectrum is strongly coupled to the bulk velocity spectrum at MHD scales (they are basically indistinguishable for $\left.k_{\perp} d_{p} \lesssim 0.4\right)$, reflecting the dominance of the ideal MHD term $\left(|\boldsymbol{u} \times \boldsymbol{B}| \sim B_{0} u_{\perp}\right)$ in the generalized Ohm's law, and is consistent with solar wind observations (Chen et al. 2011a). At smaller scales, it decouples from the velocity spectrum since the Hall term $(\boldsymbol{J} \times \boldsymbol{B} / n)$ and the electron pressure gradient term $\left(\nabla P_{e} / n\right)$ start to dominate.

Since both other fields and derivatives enter in its computation, $\boldsymbol{E}$ is the field that is mostly affected by numerical effects, and it is not straightforward to give a simple estimate of its noise level, as done for the velocity field. Ultimately, we can reasonably claim that the shallower slope of its spectrum for $2 \lesssim k_{\perp} d_{p} \lesssim 7$ is of a physical nature, while its behavior at
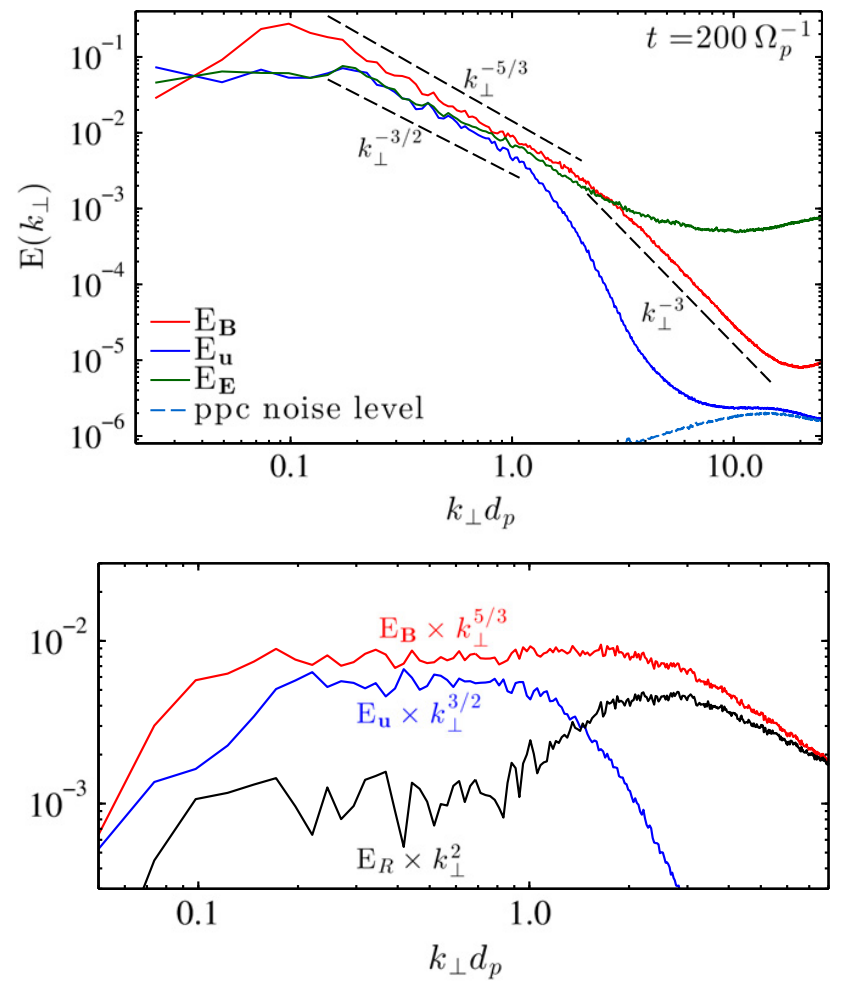

Figure 3. Top panel: omnidirectional spectra of total magnetic (red), total kinetic (blue), and total electric field (green) fluctuations vs. perpendicular wavenumber $k_{\perp}$ at $t=200 \Omega_{p}^{-1}$. The spectrum of kinetic energy at $t=0$ is plotted with a dashed light blue line as an indicator of ppc noise level. Dashed black lines are references for the corresponding spectral indices. Bottom panel: magnetic (red), kinetic (blue), and residual energy (black) spectra compensated by $k_{\perp}^{5 / 3}, k_{\perp}^{3 / 2}$, and $k_{\perp}^{2}$, respectively.

smaller scales is most likely not. On the contrary, quantitative results for the spectra of magnetic and density fluctuations are more robust, even at larger wavenumbers. A detailed description and discussion about different sources of numerical noise, e.g., the finite number of ppc, will be given in a companion paper (L. Franci et al. 2015, in preparation). For the purpose of this Letter, what matters is that such numerical noise does not affect either the qualitative scaling of the electric field spectrum for $k_{\perp} d_{p} \lesssim 7$ or the estimate of the spectral indices of other fields up to $k_{\perp} d_{p} \sim 10$ (except the velocity field, which is presumably affected by the noise level at $k_{\perp} d_{p} \gtrsim 4$ ).

The transition from the MHD regime to the sub-proton regime is not only characterized by a change in the spectral indices, but also by an increase of energy of the parallel magnetic field and the density fluctuations relative to other fields. These are shown in Figure 4, along with the parallel and the perpendicular electric field spectrum. The density and parallel magnetic fluctuations are coupled in the whole range of scales. In the MHD range, they have a flat spectrum that is an order of magnitude smaller than the perpendicular electric field. This also results in a small power in the spectrum of the total magnetic field intensity $E_{|B|}<E_{B}$ (not shown), which is consistent with solar wind observations (Horbury \& Balogh 2001). In the sub-proton range, $E_{B \|}$ and $E_{n}$ steepen, both having a clear power-law scaling with an index of -2.8 . By comparing Figures 3 and 4, one can see that the parallel and perpendicular components of magnetic fluctuations become 


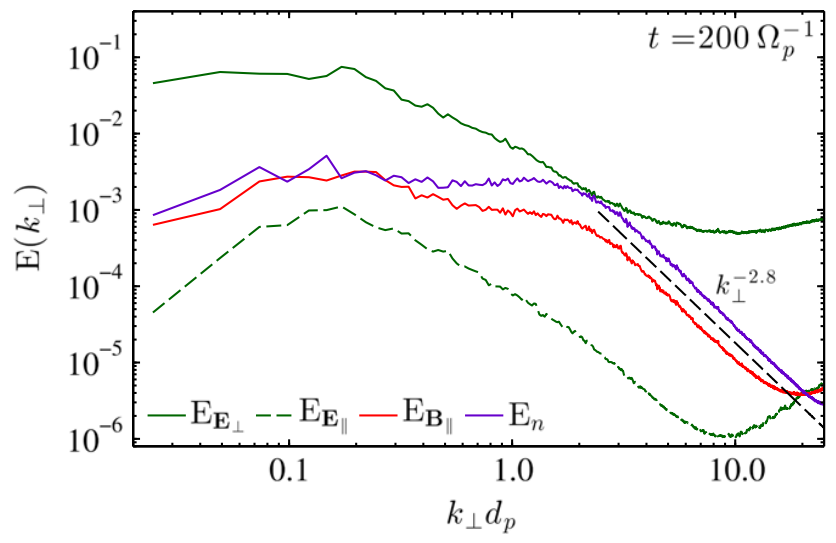

Figure 4. Omnidirectional spectra of density (purple line), parallel magnetic field (red line), parallel and perpendicular electric field (green dashed and solid lines, respectively) vs. perpendicular wavenumber $k_{\perp}$ at $t=200 \Omega_{p}^{-1}$.

comparable at the sub-proton scales, also leading to $E_{|B|} \sim E_{B}$. Concerning the electric field spectrum, at all scales, the perpendicular component $E_{E \perp}$ dominates the parallel component $E_{E \|}$ by a factor of $\sim 100$, reflecting the fact that in our configuration the leading terms of the generalized Ohm's law are linear and quadratic in the fluctuations' amplitude for $E_{E \perp}$ and $E_{E \|}$, respectively. Note that $E_{E \perp}$ flattens at the sub-proton scales and $E_{E \|}$ steepens in qualitative agreement with observations (Mozer \& Chen 2013). It is hard to determine the spectral index of $E_{E \perp}$ at sub-proton scales; a rough estimate gives $\propto k_{\perp}^{-0.8}$, consistent with $\boldsymbol{E}$ being determined by the Hall and pressure terms. In fact, retaining only the leading order in the expression of $\boldsymbol{E}$ one gets $E_{E} \sim E_{E \perp} \propto k_{\perp}^{2} E_{B_{\|}, n} \sim k_{\perp}^{-0.8}$.

We can further compare our results with observations considering three non-dimensional ratios involving density, magnetic, and electric field fluctuations shown in Figure 5. Consider first the magnetic compressibility, the ratio of parallel to total magnetic fluctuations (red line). It is negligible in the MHD range, increases while approaching the sub-proton scales, and finally saturates to a level of $\delta B_{||} / \delta B \sim 0.5$. Thus, magnetic fluctuations have mainly perpendicular components in the MHD range but tend to become isotropic at small scales, approaching a value of $\delta B_{\|}^{2} \sim \delta B_{\perp}^{2} / 3$, which is within the range $(\sim 0.2 \div 0.5)$ measured in the solar wind at spacecraft frequencies larger then $1 \mathrm{~Hz}$ (Kiyani et al. 2013). This is also in very good agreement with the level of magnetic compressibility expected for kinetic Alfvén wave turbulence for the parameters adopted in our simulation (e.g., Boldyrev et al. 2013).

The purple line in Figure 5 shows the ratio of squared normalized density fluctuations over squared normalized perpendicular magnetic fluctuations, $\delta \tilde{n}^{2} / \delta \tilde{B}_{\perp}^{2}$, where $\delta \tilde{B}_{\perp}=\delta B_{\perp} / B_{0}$ and $\delta \tilde{n}=\Gamma \delta n / n_{o}$, respectively, and $\Gamma(3 / 4$ in our simulation) is a non-dimensional kinetic normalization that depends on $T_{p}, T_{e}, \beta_{p}, v_{\mathrm{A}}$ (Schekochihin et al. 2009; Boldyrev et al. 2013). With this normalization, $\delta B_{\perp}$ and $\delta n$ are expected to have the same amplitude for kinetic Alfvénic fluctuations. Indeed, $\delta \tilde{n}^{2} / \delta \tilde{B}_{\perp}^{2}$ increases and then saturates at a value of $\sim 1$ at sub-proton scales. Note that the plateau and its value $\sim 1$ are consistent with observations (on average, $\delta \tilde{n} / \delta \tilde{B}_{\perp}=0.75$; cf. Chen et al. 2013b).

Finally, we plot the ratio between the perpendicular electric fluctuations (normalized by the Alfvén speed) and the

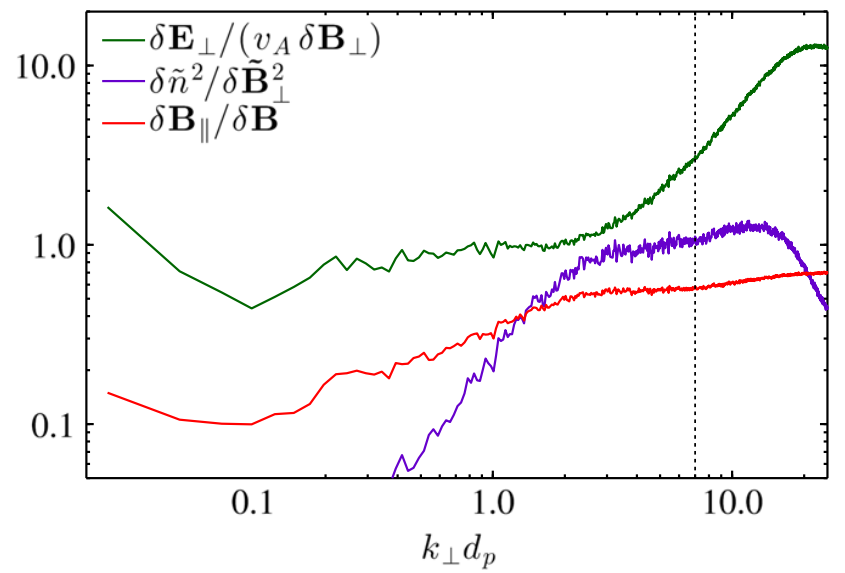

Figure 5. Ratio of perpendicular electric field to perpendicular magnetic field (green), ratio between normalized density and perpendicular magnetic fluctuations (purple; see the text for the normalizations), and ratio of parallel to total magnetic field fluctuations (magnetic compressibility; red). The numerical noise affects the ratios for $k_{\perp} d_{p} \gtrsim 7$ (vertical dotted line).

perpendicular magnetic fluctuations (green line). Similarly to the observed frequency spectra in the solar wind frame (Bale et al. 2005), this ratio is about 1 in the MHD range, where the MHD term $(\boldsymbol{u} \times \boldsymbol{B})$ dominates. At $k_{\perp} d_{p} \sim 1$, the ratio increases, reflecting the role of the Hall term $(\boldsymbol{J} \times \boldsymbol{B} / n)$ and the pressure gradient term $\left(\nabla P_{e} / n\right)$ in the generalized Ohm's law.

\section{CONCLUSION}

In this Letter, we show that hybrid 2D large-scale, highresolution simulations of turbulence are able to simultaneously reproduce several aspects of the MHD range and of the subproton range of solar wind spectra.

Two noticeable examples are given by the spectra of the magnetic field and of the electric field. The former displays a clear double power-law scaling, with spectral indices $-5 / 3$ and -3 in the MHD and sub-proton range, respectively, separated by a smooth break at $k_{\perp} d_{p} \sim 2$. The electric field spectrum also shows a change in the spectral properties at about the same scales, coupled to velocity fluctuations in the MHD range, and becomes shallower at sub-proton scales. It is also worth noting that in the MHD range we found the scaling observed in the solar wind for the magnetic, kinetic, and residual energy spectra $(-5 / 3,-3 / 2$, and -2 , respectively). To our knowledge, this is the first time that these spectral indices have been obtained for turbulence with vanishing correlation between magnetic and velocity fields. DNSs of incompressible MHD usually capture only the scaling of the residual energy and the total energy (Muller \& Grappin 2005), while reduced MHD fails in reproducing velocity and kinetic spectral indices (Chen et al. 2011b) or requires special driving (Boldyrev et al. 2011). This may indicate that it is necessary to go beyond the incompressible MHD approximation even in the inertial range. Further work is needed to test this possibility, e.g., extending the analysis to a full $3 \mathrm{D}$ simulation.

In the sub-proton scales, we found an increase in magnetic compressibility and a strong coupling between density and the parallel component of magnetic fluctuations-both having the same spectral index of -2.8 - with the main cascade of $E_{B_{\perp}}$ driven from the MHD scales. All these spectral indices match 
or are consistent with observations. The only relevant discrepancies are the flat spectra (slope $\sim 0$ ) of parallel magnetic fluctuations and density fluctuations in the MHD range. In the solar wind, they have a spectral index of $-5 / 3$ (e.g., Chen et al. 2012). This aspect is not fully captured by our simulations, which is probably because of the limited compressibility imposed by the $2 \mathrm{D}$ dynamics and/or by the value of the proton $\beta$. Note, however, that this does not prevent the full development of a compressible cascade at kinetic scales, which is in good agreement with the observations.

The features shown in Figure 5 suggest that turbulence at sub-proton scales is ruled by fluctuations with properties of kinetic Alfvén waves. However, note that the level of magnetic and gas compressibility expected for this regime follows from more general properties of the thermodynamical state assumed for the plasma $\left(\beta_{e}, \beta_{p}\right.$, and ion-electron temperature ratio), which govern the couplings between the different fields $B, E$, and $n$ via the generalized Ohm's law. In the low-frequency regime (i.e., below the whistler range), the ratios $\delta \tilde{n}^{2} / \delta \boldsymbol{B}_{\perp}^{2}$ and $\delta \boldsymbol{B}_{\|} / \delta \boldsymbol{B}$ are not expected to depend on $k$ (Boldyrev et al. 2013) since they do not rely on the specific dispersion relation of the fluctuations. In this sense, the plateaus at $2 \lesssim k_{\perp} \lesssim 7$ in Figure 5 represent a more general and likely universal manifestation of low-frequency turbulence at kinetic scales, and this is how we intend to present them here.

As a concluding remark, we stress that our simulation implements a finite resistivity to assure a source of damping at small scales for the magnetic fluctuations, and thus to prevent energy accumulation and the consequent artificial flattening of the spectrum. Although a more detailed and quantitative analysis of the related effects will be given in a forthcoming paper (Franci et al. 2015), we anticipate that the values of resistivity and the amount of ppc will affect the ion heating properties.

This project has received funding from the European Union's Seventh Framework Programme for research, technological development and demonstration under grant agreement No. 284515 (SHOCK). Website: project-shock.eu/home/. A.V. acknowledges the Interuniversity Attraction Poles Programme initiated by the Belgian Science Policy Office (IAP P7/08 CHARM). L.M. was funded by STFC grant ST/K001051/1. P.H. acknowledges GACR grant 15-10057S. H.P.C. resources were provided by CINECA (grants 2014 HP10CLF0ZB and HP10CNMQX2M). We warmly thank Frank Löffler for providing HPC resources through the Louisiana State University (allocation hpc_hyrel14).

\section{REFERENCES}

Alexandrova, O., Carbone, V., Veltri, P., \& Sorriso-Valvo, L. 2008, ApJ, 674,1153
Alexandrova, O., Lacombe, C., Mangeney, A., Grappin, R., \& Maksimovic, M. 2012, ApJ, 760, 121

Alexandrova, O., Saur, J., Lacombe, C., et al. 2009, PhRvL, 103, 165003

Bale, S. D., Kellogg, P. J., Mozer, F. S., Horbury, T. S., \& Reme, H. 2005 , PhRvL, 94, 215002

Beresnyak, A., \& Lazarian, A. 2009, ApJ, 702, 1190

Boldyrev, S., Horaites, K., Xia, Q., \& Perez, J. C. 2013, ApJ, 777, 41

Boldyrev, S., Perez, J. C., Borovsky, J. E., \& Podesta, J. J. 2011, ApJL, 741, L19

Bourouaine, S., Alexandrova, O., Marsch, E., \& Maksimovic, M. 2012, ApJ, 749,102

Bruno, R., \& Trenchi, L. 2014, ApJL, 787, L24

Camporeale, E., \& Burgess, D. 2011, ApJ, 730, 114

Chen, C. H. K., Bale, S. D., Salem, C. S., \& Maruca, B. A. 2013a, ApJ, 770,125

Chen, C. H. K., Bale, S. D., Salem, C., \& Mozer, F. S. 2011a, ApJL, 737, L41

Chen, C. H. K., Boldyrev, S., Xia, Q., \& Perez, J. C. 2013b, PhRvL, 110 225002

Chen, C. H. K., Leung, L., Boldyrev, S., Maruca, B. A., \& Bale, S. D. 2014 , GeoRL, 41, 8081

Chen, C. H. K., Mallet, A., Yousef, T. A., Schekochihin, A. A., \& Horbury, T. S. 2011b, MNRAS, 415, 3219

Chen, C. H. K., Salem, C. S., Bonnell, J. W., Mozer, F. S., \& Bale, S. D. 2012 PhRvL, 109, 035001

Dong, Y., Verdini, A., \& Grappin, R. 2014, ApJ, 793, 118

Gary, S. P., Chang, O., \& Wang, J. 2012, ApJ, 755, 142

Grappin, R., Leorat, J., \& Pouquet, A. 1983, A\&A, 126, 51

Grappin, R., \& Muller, W.-C. 2010, PhRvE, 82, 26406

Horbury, T. S., \& Balogh, A. 2001, JGR, 106, 15929

Howes, G. G., Tenbarge, J. M., Dorland, W., et al. 2011, PhRvL, 107, 035004

Kellogg, P. J., Bale, S. D., Mozer, F. S., Horbury, T. S., \& Reme, H. 2006, ApJ, 645, 704

Kiyani, K. H., Chapman, S. C., Sahraoui, F., et al. 2013, ApJ, 763, 10

Leamon, R. J., Smith, C. W., Ness, N. F., Matthaeus, W. H., \& Wong, H. K. 1998, JGR, 103, 4775

Lee, E., Brachet, M. E., Pouquet, A., Mininni, P. D., \& Rosenberg, D. 2010, PhRvE, 81, 16318

MacBride, B. T., Smith, C. W., \& Forman, M. A. 2008, ApJ, 679, 1644

Markovskii, S. A., \& Vasquez, B. J. 2011, ApJ, 739, 22

Maron, J., \& Goldreich, P. 2001, ApJ, 554, 1175

Mason, J., Cattaneo, F., \& Boldyrev, S. 2008, PhRvE, 77, 36403

Matthaeus, W. H., Servidio, S., \& Dmitruk, P. 2008, PhRvL, 101, 149501

Matthews, A. P. 1994, JCoPh, 112, 102

Meyrand, R., \& Galtier, S. 2013, PhRvL, 111, 264501

Mozer, F. S., \& Chen, C. H. K. 2013, ApJL, 768, L10

Muller, W.-C., \& Grappin, R. 2005, PhRvL, 95, 114502

Passot, T., Henri, P., Laveder, D., \& Sulem, P.-L. 2014, EPhJD, 68, 207

Podesta, J. J., Roberts, D. A., \& Goldstein, M. L. 2007, ApJ, 664, 543

Roberts, D. A. 2010, JGR, 115, 12101

Sahraoui, F., Goldstein, M. L., Belmont, G., Canu, P., \& Rezeau, L. 2010, PhRvL, 105, 131101

Salem, C., Mangeney, A., Bale, S. D., \& Veltri, P. 2009, ApJ, 702, 537

Salem, C. S., Howes, G. G., Sundkvist, D., et al. 2012, ApJL, 745, L9

Schekochihin, A. A., Cowley, S. C., Dorland, W., et al. 2009, ApJSS, 182, 310

Servidio, S., Valentini, F., Califano, F., \& Veltri, P. 2012, PhRvL, 108, 045001

Smith, C. W., Hamilton, K., Vasquez, B. J., \& Leamon, R. J. 2006, ApJL, $645, \mathrm{~L} 85$

Sorriso-Valvo, L., Marino, R., Carbone, V., et al. 2007, PhRvL, 99, 115001

Tessein, J. A., Smith, C. W., MacBride, B. T., et al. 2009, ApJ, 692, 684

Wan, M., Matthaeus, W. H., Karimabadi, H., et al. 2012, PhRvL, 109, 195001

Wicks, R. T., Horbury, T. S., Chen, C. H. K., \& Schekochihin, A. A. 2011, PhRvL, 106, 045001 